\title{
SECOND APPROXIMATE SOLUTION OF DUFFING EQUATION WITH STRONG NONLINEARITY BY HOMOTOPY PERTURBATION METHOD
}

\author{
M. Alhaz Uddin and M. Abdus Sattar \\ Department of Mathematics \\ Rajshahi University, Rajshahi-6205, Bangladesh \\ E-mail: alhazuddin@yahoo.com
}

Received 15.03.09 Accepted 17.07.10

\begin{abstract}
In this paper, the second order approximate solution of a general second order nonlinear ordinary differential system, modeling damped oscillatory process is considered. The new analytical technique based on the work of He's homotopy perturbation method is developed to find the periodic solution of a second order ordinary nonlinear differential system with damping effects. Usually the second or higher order approximate solutions are able to give better results than the first order approximate solutions. The results show that the analytical approximate solutions obtained by homotopy perturbation method are uniformly valid on the whole solutions domain and they are suitable not only for strongly nonlinear systems, but also for weakly nonlinear systems. Another advantage of this new analytical technique is that it also works for strongly damped, weakly damped and undamped systems. Figures are provided to show the comparison between the analytical and the numerical solutions.
\end{abstract}

Keywords: Homotopy perturbation method; damped oscillation; nonlinear equation; strong nonlinearity.

\section{Introduction}

The study of nonlinear differential system is of great interest in engineering and physical sciences and many other branches of applied mathematics. The solutions of nonlinear problems are very complicated and in general, it is more difficult to get an analytical approximation than a numerical one to a given nonlinear problem. There exists a wide body of literature dealing with the problem of approximate solutions to nonlinear differential equations with various different methodologies. Many different approaches have been proposed, such as Struble's techniques [5,16], Kryloff-BogoliuboffMitropolskii (KBM) [6,10] method, multiple time-scales [14] procedure, the modified Lindstedt-Poincare method [8], He's homotopy perturbation method [9], etc. Most of these methods have been originally formulated to get the periodic solution of second order nonlinear differential systems for weak or strong nonlinearity without considering any damping effects in the following form:

$$
\ddot{x}+\omega_{0}^{2} x=-\varepsilon f(x, \dot{x}), \varepsilon<<1, \varepsilon>0 .
$$

Several authors have extended these methods to investigate similar nonlinear problems with strong linear damping effects $-2 k \dot{x}, k=O(1)$ and $k>0$ modeled by the 
following equation:

$$
\ddot{x}+2 k \dot{x}+\omega^{2} x=-\varepsilon f(x, \dot{x}), \varepsilon<<1, \varepsilon>0 .
$$

Popov [15] was well known among them. He extended the KBM method and investigated the under-damped case of Eq. (2).Then Mendelson [11] reproduced Popov's results. Bojadziev [7] investigated a third order nonlinear problem with internal friction and relaxation based on the KBM technique. Following Popov [15], Murty et al. [12] investigated the over damped case of Eq. (2).They used Popov's formula by replacing the trigonometric functions with the corresponding hyperbolic functions. In their investigation, they also examined a fourth order over-damped system. Murty [13] presented a unified method for solving Eq. (2). Such a unified solution is a general one and covers the three cases viz. under-damped, undamped and over-damped situations. It is seen that the unified solution represents the original KBM solution $[6,10]$ as the limit $k \rightarrow 0$. Alam [2] has generalized Murty's [13] technique for solving an $n t h, n=2,3 \ldots$. order nonlinear differential equation. Recently, Alam et al. [5] have presented a generalized Struble's technique for solving an nth order weakly nonlinear differential system with damping effect. Thus, we observe that a considerable amount of research activities have been carried out by several authors [1-7,10-16] for the solution of the damped nonlinear systems with small nonlinearity. Therefore, the small parameter plays a very important role in the perturbation method. It determines not only the accuracy of the perturbation approximations, but also the validity of the perturbation technique itself. In Ref. [8,9], He has presented Modified Lindstedt-Poincare method for some strongly non-linear oscillations and the homotopy perturbation method for some strongly nonlinear oscillations without damping effects. But in science and engineering, there exist many nonlinear problems in presence of damping effects which do not contain any small parameter, especially those with strong nonlinearity. Thus, it is necessary to develop and improve some nonlinear analytical techniques which are independent of small parameters. The main goal of this article is to find the second order approximate solution for a general nonlinear system with strong nonlinearity in presence of damping effects. The method has been illustrated by applying it to a typical nonlinear problem of practical importance. To get our desired result, we have re-written Eq. (2) in the following form:

$$
\ddot{x}+2 k \dot{x}+\left(\omega^{2}+\varepsilon_{1}\right) x=\varepsilon\left(\frac{\varepsilon_{1}}{\varepsilon} x-f(x, \dot{x})\right), \quad \varepsilon>0,
$$

where $\varepsilon$ is a positive parameter which measures the strength of nonlinearity of the system, $\varepsilon_{1}$ is an artificial constant, $0 \leq \varepsilon_{1} \leq 1$, the significant damping term is expressed by the linear term $2 k \dot{x}$. The damping coefficient $2 k$ which is of the order of unity and also the nonlinear frequency $\omega$ of the system are constants. The assumption $\omega^{2}>k^{2}$ or $\omega^{2}<k^{2}$ guarantees the oscillating or non-oscillating character of the systems. In the most of the nonlinear dynamical systems, the quantity $\varepsilon$ is small compared with $\omega^{2}$ and its solutions may be shown to converge with the numerical results. 


\section{The Method}

In this paper, we are going to consider a general second order nonlinear ordinary differential equation in the following form:

$$
\ddot{x}+c_{1} \dot{x}+\left(c_{2}+\varepsilon_{1}\right) x=\varepsilon\left(\frac{\varepsilon_{1}}{\varepsilon} x+f(x, \dot{x})\right),
$$

where over dots denote derivatives with respect to time $t, \varepsilon$ is a positive parameter which plays an important role to the nonlinear systems, $\varepsilon_{1}$ is an artificial constant, $0 \leq \varepsilon_{1} \leq 1$, the coefficients $c_{j}, j=1,2$ are constants and $f$ is a given nonlinear function.

When $\varepsilon \rightarrow 0$, then the corresponding linear equation of Eq. (4) has two eigen values, say $\lambda_{j}, j=1,2$. Hence the general solution of the unperturbed equation of Eq. (4) leads to

$$
x(t, 0)=\sum_{j=1}^{2} a_{j} e^{\lambda_{j} t}
$$

where $a_{j}, j=1,2$ are arbitrary constants. For $\varepsilon \neq 0$, we are seeking an approximate solution of Eq. (4) in the following form:

$$
x(t, \varepsilon)=\sum_{j=1}^{2} a_{j} e^{\lambda_{j} t}+\varepsilon u_{1}(t, \varepsilon)+\varepsilon^{2} u_{2}(t, \varepsilon)+\cdots
$$

According to both Struble's $[5,16]$ technique and KBM $[6,10]$ method, the solution Eq. (6) is differentiated twice with respect to time $t$, to obtain the derivatives of $x$, i.e., $\dot{x}$ and $\ddot{x}$. Then inserting the values of $\dot{x}, \ddot{x}$ together with $x$ into Eq. (4) and after simplifying one obtains a needful formula. Clearly, this is a very difficult and tedious task. On the basis of mathematical induction, Alam [2] has presented such a general formula in terms of the variables $a_{j}(t), j=1,2, \cdots n$, for determining the KBM type solution. Further, Alam [4] has investigated a simple technique to derive the noted general formula. In this paper, we are going to present the generalized formula for the second order nonlinear differential systems with strong nonlinearity in presence of significant damping effects. This formula is used arbitrarily for the different damping effects. Now the Eq. (4) can be re-written as

$$
\prod_{j=1}^{2}\left(D-\lambda_{j}\right) x=\varepsilon\left(\frac{\varepsilon_{1}}{\varepsilon} x+f\right), \quad D \equiv \frac{d}{d t} .
$$

By substituting Eq. (6) into Eq. (7), we obtain 


$$
\prod_{j=1}^{2}\left(D-\lambda_{j}\right)\left(\sum_{j=1}^{2} a_{j} e^{\lambda_{j} t}+\varepsilon u_{1}+\varepsilon^{2} u_{2}+\cdots\right)=\varepsilon\left(\frac{\varepsilon_{1}}{\varepsilon} x+f\right),
$$

or

$\sum_{j=1}^{2}\left(\prod_{k=1, k \neq j}^{2}\left(D-\lambda_{k}\right)\right)\left(\left(D-\lambda_{j}\right) a_{j} e^{\lambda_{j} t}\right)+\prod_{j=1}^{2}\left(D-\lambda_{j}\right)\left(\varepsilon u_{1}+\varepsilon^{2} u_{2}+\cdots\right)=\varepsilon\left(\frac{\varepsilon_{1}}{\varepsilon} x+f\right)$

or

$\sum_{j=1}^{2}\left(\prod_{k=1, k \neq j}^{2}\left(D-\lambda_{k}\right)\left(\dot{a}_{j} e^{\lambda_{j} t}\right)+\prod_{j=1}^{2}\left(D-\lambda_{j}\right)\left(\varepsilon u_{1}+\varepsilon^{2} u_{2}+\cdots\right)=\varepsilon\left(\frac{\varepsilon_{1}}{\varepsilon} x+f\right)\right.$,

since $\left(D-\lambda_{j}\right)\left(a_{j} e^{\lambda_{j} t}\right)=\dot{a}_{j} e^{\lambda_{j} t}$.

\section{Example}

As an example of the above procedure, let us consider the following autonomous nonlinear differential equation:

$$
\ddot{x}+2 k \dot{x}+\omega^{2} x=-\varepsilon x^{3} .
$$

The Eq. (9) occurs in the theory of nonlinear vibrating systems and in a certain type of nonlinear electrical circuit theory. We have re-written Eq. (9) in the following form:

$$
\ddot{x}+2 k \dot{x}+\left(\omega^{2}+\varepsilon_{1}\right) x=\varepsilon\left(\frac{\varepsilon_{1}}{\varepsilon} x-x^{3}\right) .
$$

In particular, when $\varepsilon \rightarrow 0$ from Eq. (10) one obtains the unperturbed equation $\ddot{x}+2 k \dot{x}+\left(\omega^{2}+\varepsilon_{1}\right) x=0$ with two eigen-values, say $\lambda_{1,2}=-k \pm i \omega_{0}$, where $\omega_{0}=\sqrt{\left(\omega^{2}+\varepsilon_{1}-k^{2}\right)}$ or $\lambda_{1,2}=-k \pm \omega_{0}$, where $\omega_{0}=\sqrt{k^{2}-\left(\omega^{2}+\varepsilon_{1}\right)}$. Here $\omega_{0}$ is known as the reduced frequency of the system and the physical character of the motion depend on the nature of it. Thus depending on the values of $k$ and $\left(\omega^{2}+\varepsilon_{1}\right)$, the solution becomes under-damped, over-damped or critically damped. However, we are able to find a general solution in terms of the variables $a_{1}$ and $a_{2}$ as well as of the eigenvalues $\lambda_{1}$ and $\lambda_{2}$. Then by putting the values of $\lambda_{1}$ and $\lambda_{2}$, the desired solution can be found for all real or complex values of $\lambda_{1}$ and $\lambda_{2}$. Then the solution of the linearized equation of Eq. (10) is obtained by

$$
x(t, 0)=a_{1} e^{\lambda_{1} t}+a_{2} e^{\lambda_{2} t},
$$


where $a_{1}$ and $a_{2}$ are arbitrary constants. When $\varepsilon \neq 0$, we seek a general solution of Eq. (10) in the following form:

$$
x(t, \varepsilon)=a_{1} e^{\lambda_{1} t}+a_{2} e^{\lambda_{2} t}+\varepsilon u_{1}\left(a_{1}, a_{2}, t\right)+\varepsilon^{2} u_{2}\left(a_{1}, a_{2}, t\right)+\varepsilon^{3} \cdots,
$$

Here

$$
\begin{aligned}
f(x)= & \varepsilon_{1} x-\varepsilon x^{3}=\varepsilon_{1}\left(a_{1} e^{\lambda_{1} t}+a_{2} e^{\lambda_{2} t}+\varepsilon u_{1}\right)-\varepsilon\left(a_{1} e^{\lambda_{1} t}+a_{2} e^{\lambda_{2} t}+\varepsilon u_{1}\right)^{3} \\
= & \varepsilon_{1}\left(a_{1} e^{\lambda_{1} t}+a_{2} e^{\lambda_{2} t}+\varepsilon u_{1}\right)-\varepsilon\left(a_{1}^{3} e^{3 \lambda_{1} t}+3 a_{1}^{2} a_{2} e^{\left(2 \lambda_{1}+\lambda_{2}\right) t}\right. \\
& \left.+3 a_{1} a_{2}^{2} e^{\left(\lambda_{1}+2 \lambda_{2}\right) t}+a_{2}^{3} e^{3 \lambda_{2} t}+3 \varepsilon u_{1}\left(a_{1} e^{\lambda_{1} t}+a_{2} e^{\lambda_{2} t}\right)^{2}+\cdots\right) .
\end{aligned}
$$

Eq. (10) can be re-written as

$$
\begin{aligned}
(D- & \left.\lambda_{2}\right)\left(\dot{a}_{1} e^{\lambda_{1} t}\right)+\left(D-\lambda_{1}\right)\left(\dot{a}_{2} e^{\lambda_{2} t}\right)+\left(D-\lambda_{1}\right)\left(D-\lambda_{2}\right)\left(\varepsilon u_{1}+\varepsilon^{2} u_{2}+\cdots\right) \\
= & \varepsilon_{1}\left(a_{1} e^{\lambda_{1} t}+a_{2} e^{\lambda_{2} t}+\varepsilon u_{1}\right)-\varepsilon\left(a_{1} e^{\lambda_{1} t}+a_{2} e^{\lambda_{2} t}+\varepsilon u_{1}\right)^{3} \\
= & \varepsilon_{1}\left(a_{1} e^{\lambda_{1} t}+a_{2} e^{\lambda_{2} t}+\varepsilon u_{1}\right)-\varepsilon\left(a_{1}^{3} e^{3 \lambda_{1} t}+3 a_{1}^{2} a_{2} e^{\left(2 \lambda_{1}+\lambda_{2}\right) t}\right. \\
& \left.+3 a_{1} a_{2}^{2} e^{\left(\lambda_{1}+2 \lambda_{2}\right) t}+a_{2}^{3} e^{3 \lambda_{2} t}+3 \varepsilon u_{1}\left(a_{1} e^{\lambda_{1} t}+a_{2} e^{\lambda_{2} t}\right)^{2}+\cdots\right) .
\end{aligned}
$$

Now we are going to consider the terms up to $O(\varepsilon)$. According to the separation rules (details can be found in [5]), we can equate the various terms of Eq. (14) and we get the following equations:

$$
\begin{aligned}
& \left(D-\lambda_{2}\right)\left(\dot{a}_{1} e^{\lambda_{1} t}\right)=\varepsilon_{1} a_{1} e^{\lambda_{1} t}-3 \varepsilon a_{1}^{2} a_{2} e^{\left(2 \lambda_{1}+\lambda_{2}\right) t}, \\
& \left(D-\lambda_{1}\right)\left(\dot{a}_{2} e^{\lambda_{2} t}\right)=\varepsilon_{1} a_{2} e^{\lambda_{2} t}-3 \varepsilon a_{1} a_{2}^{2} e^{\left(\lambda_{1}+2 \lambda_{2}\right) t} .
\end{aligned}
$$

This leaves the following perturbational equation

$$
\left(D-\lambda_{1}\right)\left(D-\lambda_{2}\right) u_{1}=-\left(a_{1}^{3} e^{3 \lambda_{1} t}+a_{2}^{3} e^{3 \lambda_{2} t}\right) .
$$

To determine the first order approximate solution, it can be considered that $a_{1}$ and $a_{2}$ are constants. Hence the particular solutions of Eqs. (15)- (17) yield,

$$
\begin{aligned}
& \dot{a}_{1}=\varepsilon_{1} l_{0} a_{1}+\varepsilon l_{1} a_{1}^{2} a_{2} e^{\left(\lambda_{1}+\lambda_{2}\right) t}, \\
& \dot{a}_{2}=\varepsilon_{1} l_{0}^{*} a_{2}+\varepsilon l_{1}^{*} a_{1} a_{2}^{2} e^{\left(\lambda_{1}+\lambda_{2}\right) t},
\end{aligned}
$$

where

$$
l_{0}=\frac{1}{\left(\lambda_{1}-\lambda_{2}\right)}, l_{1}=-\frac{3}{2 \lambda_{1}}, l_{0}^{*}=\frac{1}{\left(\lambda_{2}-\lambda_{1}\right)}, l_{1}^{*}=-\frac{3}{2 \lambda_{2}},
$$

and

$$
u_{1}=C_{1} a_{1}^{3} e^{3 \lambda_{1} t}+C_{1}^{*} a_{2}^{3} e^{3 \lambda_{2} t},
$$

where 


$$
C_{1}=-\frac{1}{2 \lambda_{1}\left(3 \lambda_{1}-\lambda_{2}\right)}, C_{1}^{*}=-\frac{1}{2 \lambda_{2}\left(3 \lambda_{2}-\lambda_{1}\right)}
$$

Now if we substitute the values of $u_{1}$ from Eq. (19) into Eq. (14) and then simplifying, we obtain

$$
\begin{aligned}
& \left(D-\lambda_{2}\right)\left(\dot{a}_{1} e^{\lambda_{1} t}\right)+\left(D-\lambda_{1}\right)\left(\dot{a}_{2} e^{\lambda_{2} t}\right)+\varepsilon\left(D-\lambda_{1}\right)\left(D-\lambda_{2}\right)\left(C_{1} a_{1}^{3} e^{3 \lambda_{1} t}+C_{1}^{*} a_{2}^{3} e^{3 \lambda_{2} t}\right) \\
& +\left(D-\lambda_{1}\right)\left(D-\lambda_{2}\right)\left(\varepsilon^{2} u_{2}+\cdots\right) \\
& \quad=\varepsilon_{1}\left(a_{1} e^{\lambda_{1} t}+a_{2} e^{\lambda_{2} t}+\varepsilon u_{1}\right)-\varepsilon\left(a_{1}^{3} e^{3 \lambda_{1} t}+3 a_{1}^{2} a_{2} e^{\left(2 \lambda_{1}+\lambda_{2}\right) t}+3 a_{1} a_{2}^{2} e^{\left(\lambda_{1}+2 \lambda_{2}\right) t}\right. \\
& \left.\quad+a_{2}^{3} e^{3 \lambda_{2} t}\right)-3 \varepsilon^{2}\left(a_{1} e^{\lambda_{1} t}+a_{2} e^{\lambda_{2} t}\right)^{2}\left(C_{1} a_{1}^{3} e^{3 \lambda_{1} t}+C_{1}^{*} a_{2}^{3} e^{3 \lambda_{2} t}\right)
\end{aligned}
$$

Eq. (20) can be written as

$$
\begin{aligned}
& \left(D-\lambda_{2}\right)\left(\dot{a}_{1} e^{\lambda_{1} t}\right)+\left(D-\lambda_{1}\right)\left(\dot{a}_{2} e^{\lambda_{2} t}\right)+2 \varepsilon \lambda_{1}\left(3 \lambda_{1}-\lambda_{2}\right) C_{1} a_{1}^{3} e^{3 \lambda_{1} t} \\
& +2 \varepsilon \lambda_{2}\left(3 \lambda_{2}-\lambda_{1}\right) C_{1}^{*} a_{2}^{3} e^{3 \lambda_{2} t}-27 \varepsilon^{2} C_{1} a_{1}^{4} a_{2} e^{\left(4 \lambda_{1}+\lambda_{2}\right) t}-27 \varepsilon^{2} C_{1}^{*} a_{1} a_{2}^{2} e^{\left(\lambda_{1}+4 \lambda_{2}\right) t} \\
& +3 \varepsilon \varepsilon_{1}\left(5 \lambda_{1}-\lambda_{2}\right) l_{0} C_{1} a_{1}^{3} e^{3 \lambda_{1} t}+3 \varepsilon \varepsilon_{1}\left(5 \lambda_{2}-\lambda_{1}\right) l_{0}^{*} C_{1}^{*} a_{2}^{3} e^{3 \lambda_{2} t} \\
& +\left(D-\lambda_{1}\right)\left(D-\lambda_{2}\right)\left(\varepsilon^{2} u_{2}+\ldots\right) \\
& =\varepsilon_{1}\left(a_{1} e^{\lambda_{1} t}+a_{2} e^{\lambda_{2} t}\right)+\varepsilon \varepsilon_{1}\left(C_{1} a_{1}^{3} e^{3 \lambda_{1} t}+C_{1}^{*} a_{2}^{3} e^{3 \lambda_{2} t}\right) \\
& -\varepsilon\left(a_{1}^{3} e^{3 \lambda_{1} t}+3 a_{1}^{2} a_{2} e^{\left(2 \lambda_{1}+\lambda_{2}\right) t}+3 a_{1} a_{2}^{2} e^{\left(\lambda_{1}+2 \lambda_{2}\right) t}+a_{2}^{3} e^{3 \lambda_{2} t}\right) \\
& -3 \varepsilon^{2}\left(C_{1} a_{1}^{5} e^{5 \lambda_{1} t}+2 C_{1} a_{1}^{4} a_{2} e^{\left(4 \lambda_{1}+\lambda_{2}\right) t}+C_{1} a_{1}^{3} a_{2}^{2} e^{\left(3 \lambda_{1}+2 \lambda_{2}\right) t}\right. \\
& \left.+C_{1}^{*} a_{1}^{2} a_{2}^{3} e^{\left(2 \lambda_{1}+3 \lambda_{2}\right) t}+2 C_{1}^{*} a_{1} a_{2}^{4} e^{\left(\lambda_{1}+4 \lambda_{2}\right) t}+C_{1}^{*} a_{2}^{5} e^{5 \lambda_{2} t}\right) .
\end{aligned}
$$

All the terms with $\varepsilon$ of the left side and the terms $a_{1}^{3} e^{3 \lambda_{1} t}, a_{2}^{3} e^{3 \lambda_{2} t}$ of the right side of Eq. (21) are cancelled since $C_{1}$ and $C_{1}^{*}$ satisfy Eq. (19). According to the separation rules (in Ref [5]), $u_{2}$ excludes the terms $a_{1}^{3} a_{2}^{2} e^{\left(3 \lambda_{1}+2 \lambda_{2}\right) t}$ and $a_{1}^{2} a_{2}^{3} e^{\left(2 \lambda_{1}+3 \lambda_{2}\right) t}$ and they will be added to the equations of $\dot{a}_{1}$ and $\dot{a}_{2}$ respectively. Therefore, we obtain the equations for $\dot{a}_{1}$ and $\dot{a}_{2}$ up to $O\left(\varepsilon^{2}\right)$ and $u_{2}$ in the following forms:

$$
\begin{aligned}
& \left(D-\lambda_{2}\right)\left(\dot{a}_{1} e^{\lambda_{1} t}\right)=\varepsilon_{1} a_{1} e^{\lambda_{1} t}-3 \varepsilon a_{1}^{2} a_{2} e^{\left(2 \lambda_{1}+\lambda_{2}\right) t}-3 \varepsilon^{2} C_{1} a_{1}^{3} a_{2}^{2} e^{\left(3 \lambda_{1}+2 \lambda_{2}\right) t}, \\
& \left(D-\lambda_{1}\right)\left(\dot{a}_{2} e^{\lambda_{2} t}\right)=\varepsilon_{1} a_{2} e^{\lambda_{2} t}-3 \varepsilon a_{1} a_{2}^{2} e^{\left(\lambda_{1}+2 \lambda_{2}\right) t}-3 \varepsilon^{2} C_{1}^{*} a_{1}^{2} a_{2}^{3} e^{\left(2 \lambda_{1}+3 \lambda_{2}\right) t},
\end{aligned}
$$

and 


$$
\begin{aligned}
& \left(D-\lambda_{1}\right)\left(D-\lambda_{2}\right) u_{2} \\
= & 27 C_{1} a_{1}^{4} a_{2} e^{\left(4 \lambda_{1}+\lambda_{2}\right) t}+27 C_{1}^{*} a_{1} a_{2}^{4} e^{\left(\lambda_{1}+4 \lambda_{2}\right) t}-\frac{3 \varepsilon_{1}}{\varepsilon}\left(5 \lambda_{1}-\lambda_{2}\right) l_{0} C_{1} a_{1}^{3} e^{3 \lambda_{1} t} \\
& -\frac{3 \varepsilon_{1}}{\varepsilon}\left(5 \lambda_{2}-\lambda_{1}\right) l_{0}^{*} C_{1}^{*} a_{2}^{3} e^{3 \lambda_{2} t}+\frac{\varepsilon_{1}}{\varepsilon}\left(C_{1} a_{1}^{3} e^{3 \lambda_{1} t}+C_{1}^{*} a_{2}^{3} e^{3 \lambda_{2} t}\right) \\
& -3\left(C_{1} a_{1}^{5} e^{5 \lambda_{1} t}+2 C_{1} a_{1}^{4} a_{2} e^{\left(4 \lambda_{1}+\lambda_{2}\right) t}+C_{1} a_{1}^{3} a_{2}^{2} e^{\left(3 \lambda_{1}+2 \lambda_{2}\right) t}+C_{1}^{*} a_{1}^{2} a_{2}^{3} e^{\left(2 \lambda_{1}+3 \lambda_{2}\right) t}\right. \\
& \left.+2 C_{1}^{*} a_{1} a_{2}^{4} e^{\left(\lambda_{1}+4 \lambda_{2}\right) t}+C_{1}^{*} a_{2}^{5} e^{5 \lambda_{2} t}\right) .
\end{aligned}
$$

Now we are going to assume the particular solutions of Eqs. (22)- (23) in the following forms:

$$
\begin{aligned}
& \dot{a}_{1}=\varepsilon_{1} l_{0} a_{1}+\varepsilon l_{1} a_{1}^{2} a_{2} e^{\left(\lambda_{1}+\lambda_{2}\right) t}+\varepsilon^{2} l_{2} a_{1}^{3} a_{2}^{2} e^{2\left(\lambda_{1}+\lambda_{2}\right) t}+\varepsilon \varepsilon_{1} l_{3} a_{1}^{2} a_{2} e^{\left(\lambda_{1}+\lambda_{2}\right) t}+\varepsilon_{1}^{2} l_{4} a_{1}, \\
& \dot{a}_{2}=\varepsilon_{1} l_{0}^{*} a_{2}+\varepsilon l_{1}^{*} a_{1} a_{2}^{2} e^{\left(\lambda_{1}+\lambda_{2}\right) t}+\varepsilon^{2} l_{2}^{*} a_{1}^{2} a_{2}^{3} e^{2\left(\lambda_{1}+\lambda_{2}\right) t}+\varepsilon \varepsilon_{1} l_{3}^{*} a_{1} a_{2}^{2} e^{\left(\lambda_{1}+\lambda_{2}\right) t}+\varepsilon_{1}^{2} l_{4}^{*} a_{2},
\end{aligned}
$$

where $l_{0}, l_{1}, l_{0}^{*}$ and $l_{1}^{*}$ are given in Eq. (18) while $l_{2}, l_{3}, l_{4}, l_{2}^{*} l_{3}^{*}$ and $l_{4}^{*}$ are to be determined. Now substituting the values of $\dot{a}_{1}$ and $\dot{a}_{2}$ from Eq. (25) into Eqs. (22)- (23), and then simplifying, we get

$$
\begin{aligned}
& \varepsilon_{1}\left(\lambda_{1}-\lambda_{2}\right) l_{0} a_{1} e^{\lambda_{1} t}+2 \varepsilon \lambda_{1} l_{1} a_{1}^{2} a_{2} e^{\left(2 \lambda_{1}+\lambda_{2}\right) t}+\varepsilon^{2}\left\{l_{1}\left(2 l_{1}+l_{1}^{*}\right)\right. \\
& \left.+\left(3 \lambda_{1}+\lambda_{2}\right) l_{2}\right\} a_{1}^{3} a_{2}^{2} e^{\left(3 \lambda_{1}+2 \lambda_{2}\right) t}+\varepsilon \varepsilon_{1}\left\{l_{1}\left(3 l_{0}+l_{0}^{*}\right)\right. \\
& \left.+2 \lambda_{1} l_{3}\right\} a_{1}^{2} a_{2} e^{\left(2 \lambda_{1}+\lambda_{2}\right) t}+\varepsilon_{1}^{2}\left\{l_{0}^{2}+\left(\lambda_{1}-\lambda_{2}\right) l_{4}\right\} a_{1} e^{\lambda_{1} t} \\
& =\varepsilon_{1} a_{1} e^{\lambda_{1} t}-3 \varepsilon a_{1}^{2} a_{2} e^{\left(2 \lambda_{1}+\lambda_{2}\right) t}-3 \varepsilon^{2} C_{1} a_{1}^{3} a_{2}^{2} e^{\left(3 \lambda_{1}+2 \lambda_{2}\right) t},
\end{aligned}
$$

and

$$
\begin{aligned}
& \varepsilon_{1}\left(\lambda_{1}-\lambda_{2}\right) l_{0}^{*} a_{2} e^{\lambda_{2} t}+2 \varepsilon \lambda_{2} l_{1}^{*} a_{1} a_{2}^{2} e^{\left(\lambda_{1}+2 \lambda_{2}\right) t} \\
& +\varepsilon^{2}\left\{l_{1}^{*}\left(2 l_{1}^{*}+l_{1}\right)+\left(\lambda_{1}+3 \lambda_{2}\right) l_{2}^{*}\right\} a_{1}^{2} a_{2}^{3} e^{\left(2 \lambda_{1}+3 \lambda_{2}\right) t} \\
& +\varepsilon \varepsilon_{1}\left\{l_{1}^{*}\left(3 l_{0}^{*}+l_{0}\right)+2 \lambda_{2} l_{3}^{*}\right\} a_{1} a_{2}^{2} e^{\left(\lambda_{1}+2 \lambda_{2}\right) t} \\
& +\varepsilon_{1}^{2}\left\{l_{0}^{* 2}+\left(\lambda_{2}-\lambda_{1}\right) l_{4}^{*}\right\} a_{2} e^{\lambda_{2} t} \\
& =\varepsilon_{1} a_{2} e^{\lambda_{2} t}-3 \varepsilon a_{1} a_{2}^{2} e^{\left(\lambda_{1}+2 \lambda_{2}\right) t}-3 \varepsilon^{2} C_{1}^{*} a_{1}^{2} a_{2}^{3} e^{\left(2 \lambda_{1}+3 \lambda_{2}\right) t} .
\end{aligned}
$$

All the terms with $\varepsilon$ of Eqs. (26) - (27) are cancelled since $l_{0}, l_{1}, l_{0}^{*}$ and $l^{*}$ satisfy Eq. (18). By comparing the coefficients of $\varepsilon^{2}, \varepsilon \varepsilon_{1}$ and $\varepsilon_{1}^{2}$ from both sides of Eqs. (26)(27), we get the following algebraic equations: 


$$
\begin{aligned}
& l_{1}\left(2 l_{1}+l_{1}^{*}\right)+\left(3 \lambda_{1}+\lambda_{2}\right) l_{2}=-3 C_{1}, \\
& l_{1}\left(3 l_{0}+l_{0}^{*}\right)+2 \lambda_{1} l_{3}=0, \\
& l_{1}^{2}+\left(\lambda_{1}-\lambda_{2}\right) l_{4}=0,
\end{aligned}
$$

and

$$
\begin{aligned}
& l_{1}^{*}\left(2 l_{1}^{*}+l_{1}\right)+\left(\lambda_{1}+3 \lambda_{2}\right) l_{2}^{*}=-3 C_{1}^{*}, \\
& l_{1}^{*}\left(3 l_{0}^{*}+l_{0}\right)+2 \lambda_{2} l_{3}^{*}=0, \\
& l_{1}^{* 2}+\left(\lambda_{2}-\lambda_{1}\right) l_{4}^{*}=0 .
\end{aligned}
$$

By solving Eqs. (28)- (29) and then substituting the values of $l_{1}, l_{1}^{*}, C_{1}$ and $C_{1}^{*}$ from Eqs. (18)- (19) and then by simplifying, we have

$$
\begin{aligned}
& l_{2}=\frac{3\left(-9 \lambda_{2}^{2}-13 \lambda_{1} \lambda_{2}+6 \lambda_{1}^{2}\right)}{4 \lambda_{1}^{2} \lambda_{2}\left(9 \lambda_{1}^{2}-\lambda_{2}^{2}\right)}, l_{3}=\frac{3}{2 \lambda_{1}^{2}\left(\lambda_{1}-\lambda_{2}\right)}, l_{4}=-\frac{1}{\left(\lambda_{1}-\lambda_{2}\right)^{3}}, \\
& l_{2}^{*}=\frac{3\left(-9 \lambda_{1}^{2}-13 \lambda_{1} \lambda_{2}+6 \lambda_{2}^{2}\right)}{4 \lambda_{1} \lambda_{2}^{2}\left(9 \lambda_{2}^{2}-\lambda_{1}^{2}\right)}, l_{3}^{*}=\frac{3}{2 \lambda_{2}^{2}\left(\lambda_{2}-\lambda_{1}\right)}, l_{4}^{*}=-\frac{1}{\left(\lambda_{2}-\lambda_{1}\right)^{3}} .
\end{aligned}
$$

On simplification Eq. (24) gives

$$
\begin{aligned}
& \left(D-\lambda_{1}\right)\left(D-\lambda_{2}\right) u_{2} \\
= & \frac{\varepsilon_{1}}{\varepsilon}\left\{1-3\left(5 \lambda_{1}-\lambda_{2}\right) l_{0}\right\} C_{1} a_{1}^{3} e^{3 \lambda_{1} t}+\frac{\varepsilon_{1}}{\varepsilon}\left\{1-3\left(5 \lambda_{2}-\lambda_{1}\right) l_{0}^{*}\right\} C_{1}^{*} a_{2}^{3} e^{3 \lambda_{2} t} \\
& +21 C_{1} a_{1}^{4} a_{2} e^{\left(4 \lambda_{1}+\lambda_{2}\right) t}+21 C_{1}^{*} a_{1} a_{2}^{4} e^{\left(\lambda_{1}+4 \lambda_{2}\right) t}-3 C_{1} a_{1}^{5} e^{5 \lambda_{1} t}-3 C_{1}^{*} a_{2}^{5} e^{5 \lambda_{2} t} .
\end{aligned}
$$

By solving Eq. (31) for $u_{2}$, we obtain the particular solution as

$$
\begin{aligned}
u_{2}= & \frac{\varepsilon_{1}}{\varepsilon}\left\{\frac{1-3\left(5 \lambda_{1}-\lambda_{2}\right) l_{0}}{2 \lambda_{1}\left(3 \lambda_{1}-\lambda_{2}\right)}\right\} C_{1} a_{1}^{3} e^{3 \lambda_{1} t}+\frac{\varepsilon_{1}}{\varepsilon}\left\{\frac{1-3\left(5 \lambda_{2}-\lambda_{1}\right) l_{0}^{*}}{2 \lambda_{2}\left(3 \lambda_{2}-\lambda_{1}\right)}\right\} C_{1}^{*} a_{2}^{3} e^{3 \lambda_{2} t} \\
& +\frac{21 C_{1} a_{1}^{4} a_{2} e^{\left(4 \lambda_{1}+\lambda_{2}\right) t}}{4 \lambda_{1}\left(3 \lambda_{1}+\lambda_{2}\right)}+\frac{21 C_{1}^{*} a_{1} a_{2}^{4} e^{\left(\lambda_{1}+4 \lambda_{2}\right) t}}{4 \lambda_{2}\left(\lambda_{1}+3 \lambda_{2}\right)}-\frac{3 C_{1} a_{1}^{5} e^{5 \lambda_{1} t}}{4 \lambda_{1}\left(5 \lambda_{1}-\lambda_{2}\right)}-\frac{3 C_{1}^{*} a_{2}^{5} e^{5 \lambda_{2} t}}{4 \lambda_{2}\left(5 \lambda_{2}-\lambda_{1}\right)}
\end{aligned}
$$

Finally, inserting the values of $l_{0}, l_{0}^{*}, C_{1}$ and $C_{1}^{*}$ from Eqs. (18)- (19) into Eq. (32) and simplifying, we get, 


$$
\begin{aligned}
u_{2}= & \frac{\varepsilon_{1}}{\varepsilon}\left(E_{0} a_{1}^{3} e^{3 \lambda_{1} t}+E_{0}^{*} a_{2}^{3} e^{3 \lambda_{2} t}\right)+E_{2} a_{1}^{4} a_{2} e^{\left(4 \lambda_{1}+\lambda_{2}\right) t} \\
& +E_{2}^{*} a_{1} a_{2}^{4} e^{\left(\lambda_{1}+4 \lambda_{2}\right) t}+C_{2} a_{1}^{5} e^{5 \lambda_{1} t}+C_{2}^{*} a_{2}^{5} e^{5 \lambda_{2} t},
\end{aligned}
$$

where

$$
\begin{array}{ll}
E_{0}=\frac{7 \lambda_{1}-\lambda_{2}}{\left[2 \lambda_{1}^{2}\left(\lambda_{1}-\lambda_{2}\right)\left(3 \lambda_{1}-\lambda_{2}\right)^{2}\right]}, & E_{0}^{*}=\frac{7 \lambda_{2}-\lambda_{1}}{\left[2 \lambda_{2}^{2}\left(\lambda_{2}-\lambda_{1}\right)\left(3 \lambda_{2}-\lambda_{1}\right)^{2}\right.}, \\
E_{2}=-\frac{21}{\left[8 \lambda_{1}^{2}\left(9 \lambda_{1}^{2}-\lambda_{2}^{2}\right)\right]}, & E_{2}^{*}=-\frac{21}{\left[8 \lambda_{2}^{2}\left(9 \lambda_{2}^{2}-\lambda_{1}^{2}\right)\right]}, \\
C_{2}=\frac{3}{\left[8 \lambda_{1}^{2}\left(3 \lambda_{1}-\lambda_{2}\right)\left(5 \lambda_{1}-\lambda_{2}\right)\right]}, & C_{2}^{*}=\frac{3}{\left[8 \lambda_{2}^{2}\left(3 \lambda_{2}-\lambda_{1}\right)\left(5 \lambda_{2}-\lambda_{1}\right)\right]} .
\end{array}
$$

Thus, the second order approximate solution of Eq. (10) is obtained as

$$
x(t, \varepsilon)=a_{1} e^{\lambda_{1} t}+a_{2} e^{\lambda_{2} t}+\varepsilon u_{1}\left(a_{1}, a_{2}, t\right)+\varepsilon^{2} u_{2}\left(a_{1}, a_{2}, t\right),
$$

where $a_{1}$ and $a_{2}$ are the solutions of Eq. (25) and $u_{1}, u_{2}$ are respectively given by Eqs. (19) and (33).This solution can be carried out to the usual form by using the suitable transformations. For the under-damped system, the variables should be transformed by

$$
a_{1}=\frac{1}{2} a e^{i \theta}, \quad a_{2}=\frac{1}{2} a e^{-i \theta} .
$$

Now inserting Eq. (36) into Eq. (25) and simplifying them, we obtain the following variational equations for the amplitude and phase variables:

$$
\begin{aligned}
& \dot{a}=\varepsilon m_{1} a^{3} e^{-2 k t}+\varepsilon^{2} m_{2} a^{5} e^{-4 k t}+\varepsilon \varepsilon_{1} m_{3} a^{3} e^{-2 k t}, \\
& \dot{\theta}=\varepsilon_{1} n_{0}+\varepsilon n_{1} a^{2} e^{-2 k t}+\varepsilon^{2} n_{2} a^{4} e^{-4 k t}+\varepsilon \varepsilon_{1} n_{3} a^{2} e^{-2 k t}+\varepsilon_{1}{ }^{2} n_{4},
\end{aligned}
$$

where

$$
\begin{aligned}
& m_{1}=\frac{3 k}{8\left(k^{2}+\omega_{0}^{2}\right)}, m_{2}=\frac{3 k\left\{15 \omega_{0}^{2}\left(7 k^{2}-2 \omega_{0}^{2}\right)-\left(7 \omega_{0}^{2}-k^{2}\right)\left(8 k^{2}+5 \omega_{0}^{2}\right)\right\}}{128\left(k^{2}+\omega_{0}^{2}\right)^{2}\left(k^{2}+4 \omega_{0}^{2}\right)\left(4 k^{2}+\omega_{0}^{2}\right)}, \\
& m_{3}=\frac{3 k}{8\left(k^{2}+\omega_{0}^{2}\right)^{2}}, n_{0}=-\frac{1}{2 \omega_{0}}, \quad n_{1}=\frac{3 \omega_{0}}{8\left(k^{2}+\omega_{0}^{2}\right)}, \\
& n_{2}=\frac{3 \omega_{0}\left\{15 k^{2}\left(7 \omega_{0}^{2}-2 k^{2}\right)+\left(7 k^{2}-2 \omega_{0}^{2}\right)\left(8 k^{2}+5 \omega_{0}^{2}\right)\right\}}{128\left(k^{2}+\omega_{0}^{2}\right)^{2}\left(k^{2}+4 \omega_{0}^{2}\right)\left(4 k^{2}+\omega_{0}^{2}\right)}, \\
& n_{3}=-\frac{3\left(k^{2}-\omega_{0}^{2}\right)}{16 \omega_{0}\left(k^{2}+\omega_{0}^{2}\right)^{2}}, \quad n_{4}=-\frac{1}{2 \omega_{0}^{2}} .
\end{aligned}
$$


By using Eq. (36) and assuming that $\varphi=\omega_{0} t+\theta$, the correction terms $u_{1}$ and $u_{2}$ can be written in the following forms:

$$
u_{1}=a^{3} e^{-3 k t}\left(P_{3} \cos 3 \varphi+Q_{3} \sin 3 \varphi\right),
$$

and

$$
\begin{aligned}
u_{2}= & \frac{\varepsilon_{1}}{\varepsilon} a^{3} e^{-3 k t}\left(P_{4} \cos 3 \varphi+Q_{4} \sin 3 \varphi\right)+a^{5} e^{-5 k t}\left(P_{5} \cos 3 \varphi+Q_{5} \sin 3 \varphi\right) \\
& +a^{5} e^{-5 k t}\left(P_{6} \cos 5 \varphi+Q_{6} \sin 5 \varphi\right),
\end{aligned}
$$

where

$$
P_{3}=-\frac{\left(k^{2}-2 \omega_{0}^{2}\right)}{16\left(k^{2}+\omega_{0}^{2}\right)\left(k^{2}+4 \omega_{0}^{2}\right)}, \quad Q_{3}=\frac{3 k \omega_{0}}{16\left(k^{2}+\omega_{0}^{2}\right)\left(k^{2}+4 \omega_{0}^{2}\right)},
$$

and

$$
\begin{aligned}
& P_{4}=-\frac{\left(7 k^{4}+8 k^{2} \omega_{0}^{2}-8 \omega_{0}^{4}\right)}{16\left(k^{2}+\omega_{0}^{2}\right)^{2}\left(k^{2}+4 \omega_{0}^{2}\right)^{2}}, Q_{4}=-\frac{3 k\left(k^{4}-5 k^{2} \omega_{0}^{2}-12 \omega_{0}^{4}\right)}{32 \omega_{0}\left(k^{2}+\omega_{0}^{2}\right)^{2}\left(k^{2}+4 \omega_{0}^{2}\right)^{2}}, \\
& P_{5}=-\frac{21\left(k^{4}-7 k^{2} \omega_{0}^{2}+\omega_{0}^{4}\right)}{256\left(k^{2}+\omega_{0}^{2}\right)^{2}\left(k^{2}+4 \omega_{0}^{2}\right)\left(4 k^{2}+\omega_{0}^{2}\right)}, \\
& Q_{5}=\frac{189 k \omega_{0}\left(k^{2}-\omega_{0}^{2}\right)}{512\left(k^{2}+\omega_{0}^{2}\right)^{2}\left(k^{2}+4 \omega_{0}^{2}\right)\left(4 k^{2}+\omega_{0}^{2}\right)}, \\
& P_{6}=\frac{3\left(k^{4}-11 k^{2} \omega_{0}^{2}+3 \omega_{0}^{4}\right)}{256\left(k^{2}+\omega_{0}^{2}\right)^{2}\left(k^{2}+4 \omega_{0}^{2}\right)\left(4 k^{2}+9 \omega_{0}^{2}\right)}, \\
& Q_{6}=-\frac{3 k \omega_{0}\left(11 k^{2}-19 \omega_{0}^{2}\right)}{512\left(k^{2}+\omega_{0}^{2}\right)^{2}\left(k^{2}+4 \omega_{0}^{2}\right)\left(4 k^{2}+9 \omega_{0}^{2}\right)} .
\end{aligned}
$$

Hence the second order approximate solution of Eq. (10) for the damped oscillatory process is given by

$$
x=a e^{-k t} \cos \varphi+\varepsilon u_{1}+\varepsilon^{2} u_{2}, \varphi=\omega_{0} t+\theta,
$$

where $a$ and $\theta$ are the solutions of Eq. (37) and $u_{1}$ and $u_{2}$ are respectively obtained from Eqs. (39) and (40).

\section{Results and Discussion}

The obtained approximate solutions are compared with the numerical solutions graphically. Also to show the effect of second order approximate solutions, graphs are drawn for both first and second order approximations. 
In Fig.1 (a), comparison is made between the first order approximate solution and the numerical solution obtained by using Runge-Kutta fourth order formula for strong nonlinearity with large damping effects. Here we notice that with the increase of time $t$ the analytical result deviates from the numerical one. Fig.1 (b) represents the same for the second order approximate solution within the same time domain and it is observed that the deviation from the numerical result is very small in the case of second order approximate solution. In Fig.2 (a), comparison is made between the first order approximate solution and the numerical solution obtained by using Runge-Kutta fourth order formula for strong nonlinearity with small damping effects. Here we notice that with the increase of time $t$ the analytical result deviates from the numerical one. Fig.2 (b) represents the same for the second order approximate solution within the same time domain and it is observed that the deviation from the numerical result is very small in case of the second order approximate solution.

In Fig.3 (a), comparison is made between the first order approximate solution and the numerical solution obtained by using Runge-Kutta fourth order formula for strong nonlinearity without damping effect. From this figure, we notice that with the increase of time $t$ the analytical results deviate from the numerical one. Fig.3 (b) represents the same for the second order approximate solution within the same time domain and it is observed that the analytical solution has good agreement with the numerical result in the case of second order approximate solution. It may be mentioned that if we consider $\varepsilon_{1}=0$, then our result becomes the same as that of Alam et al. [5]. To check this, we have plotted the Figs.4 (a, b). In Fig.4 (a), comparison is made between the first order approximate solution and the numerical solution obtained by using Runge-Kutta fourth order formula for strong nonlinearity with large damping effects. Here we notice that with the increase of time $t$ the analytical result deviates from the numerical one. Fig.4 (b) represents the same for the second order approximate solution within the same time domain and it is observed that the deviation from the numerical result is very small in the case of second order approximate solution by setting $\varepsilon_{1}=0$. From the Figs.1 (a, b) and Figs. $4(\mathbf{a}, \mathbf{b})$, it is notified that, our new homotopy perturbation technique gives better result than that of Alam et al. [5]. 


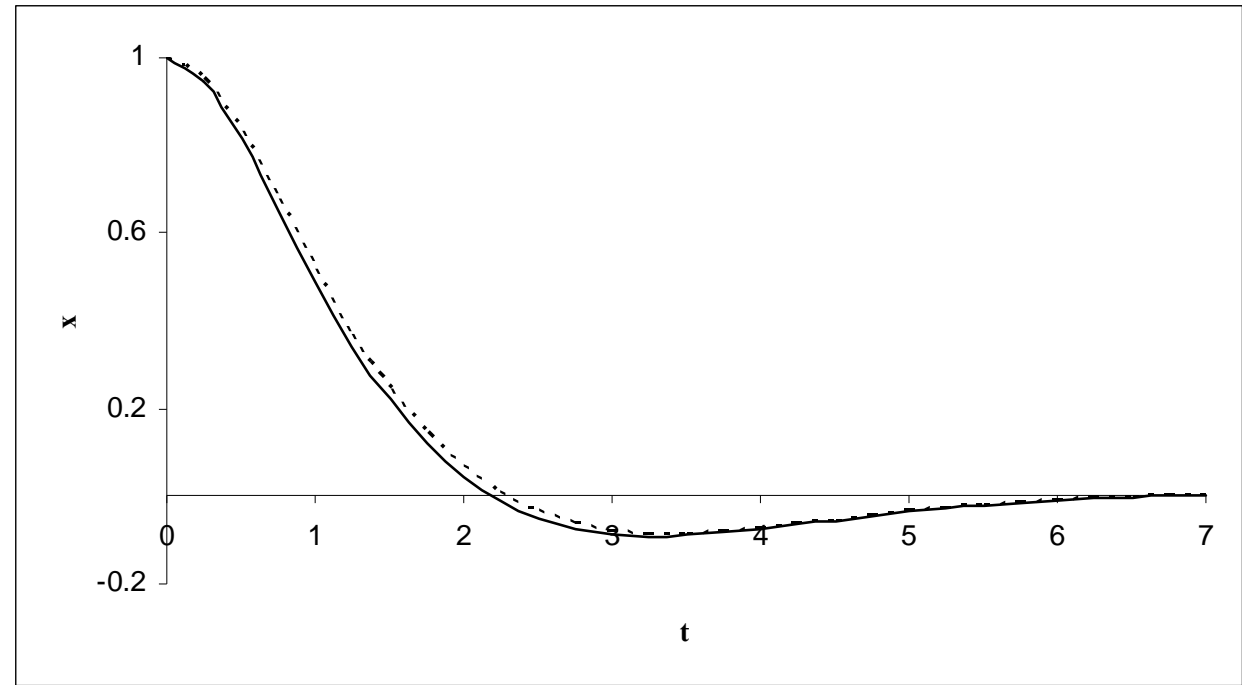

Fig.1. (a) First order approximate solution (dotted lines) of Eq. (10) is compared with the corresponding numerical solution (solid line) obtained by Runge-Kutta fourth-order formula for $a_{0}=1.07073$, $\varphi_{0}=-0.31590, k=\operatorname{sqrt}(.5), \omega=1.0, \varepsilon_{1}=0.2$ and $\varepsilon=1.0$.

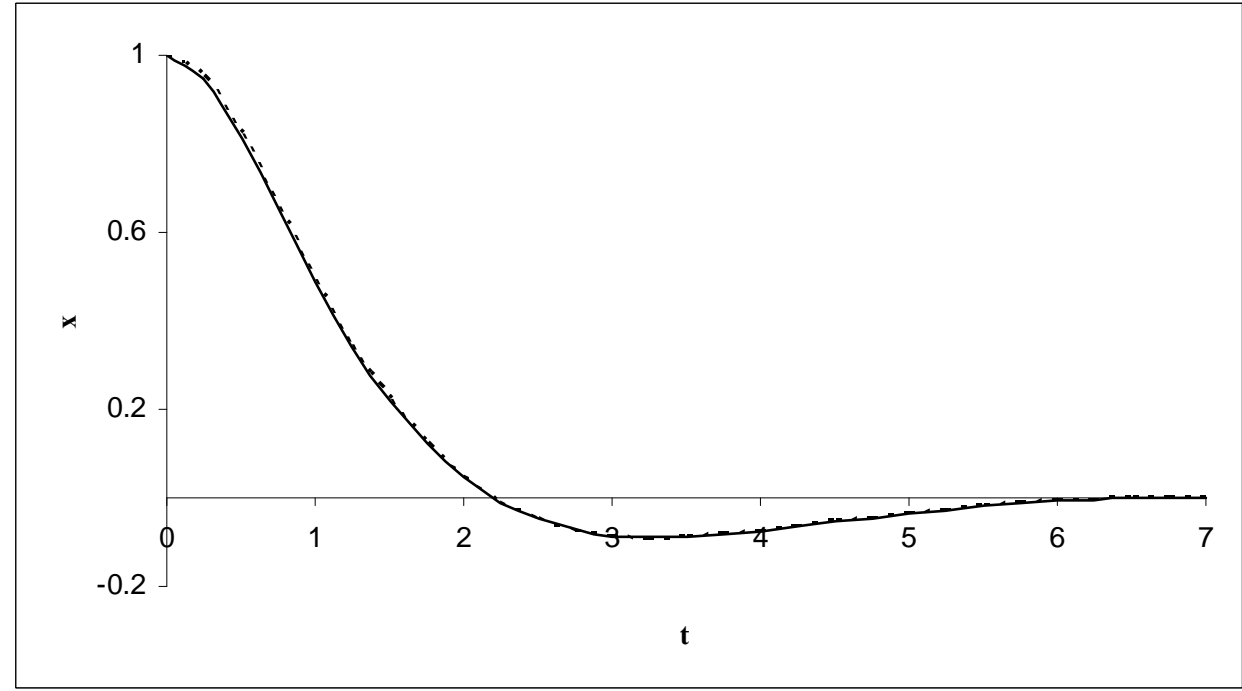

Fig.1. (b) Second order approximate solution (dotted lines) of Eq. (10) is compared with the corresponding numerical solution (solid line) obtained by Runge-Kutta fourth-order formula for $a_{0}=1.04923$, $\varphi_{0}=-0.27983, k=\operatorname{sqrt}(.5), \omega=1.0, \varepsilon_{1}=0.2$ and $\varepsilon=1.0$. 


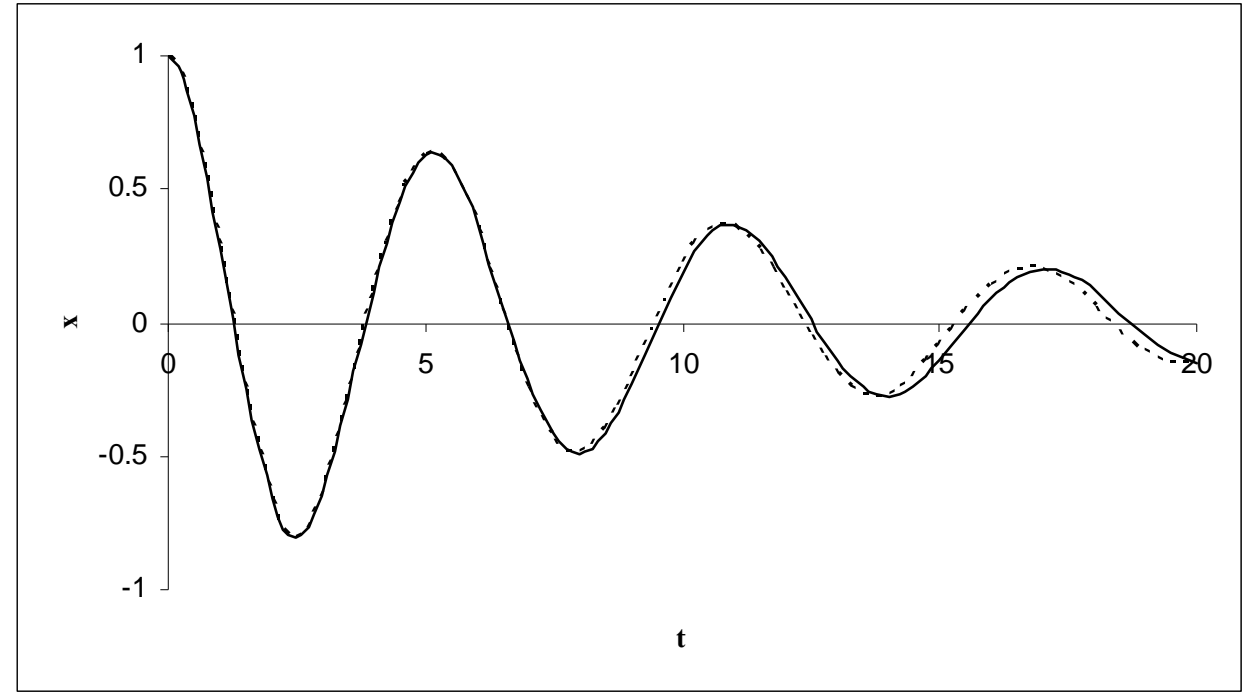

Fig.2. (a) First order approximate solution (dotted lines) of Eq. (10) is compared with the corresponding numerical solution (solid lines) obtained by Runge-Kutta fourth-order formula for $a_{0}=0.98477$, $\varphi_{0}=-0.05055, k=0.1, \omega=1.0, \varepsilon_{1}=0.75$ and $\varepsilon=1.0$.

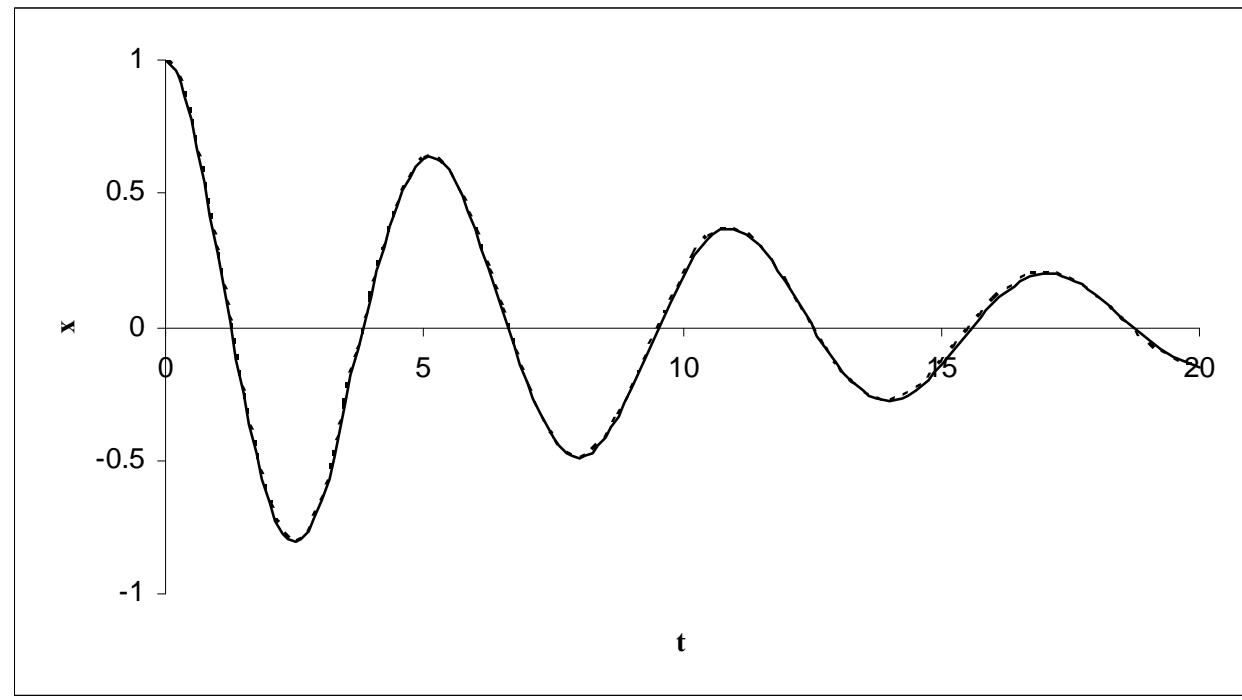

Fig.2. (b) Second order approximate solution (dotted lines) of Eq. (10) is compared with the corresponding numerical solution (solid line) obtained by Runge-Kutta fourth-order formula for $a_{0}=0.98313$, $\varphi_{0}=-0.05200, k=0.1, \omega=1.0, \varepsilon_{1}=0.75$ and $\varepsilon=1.0$. 


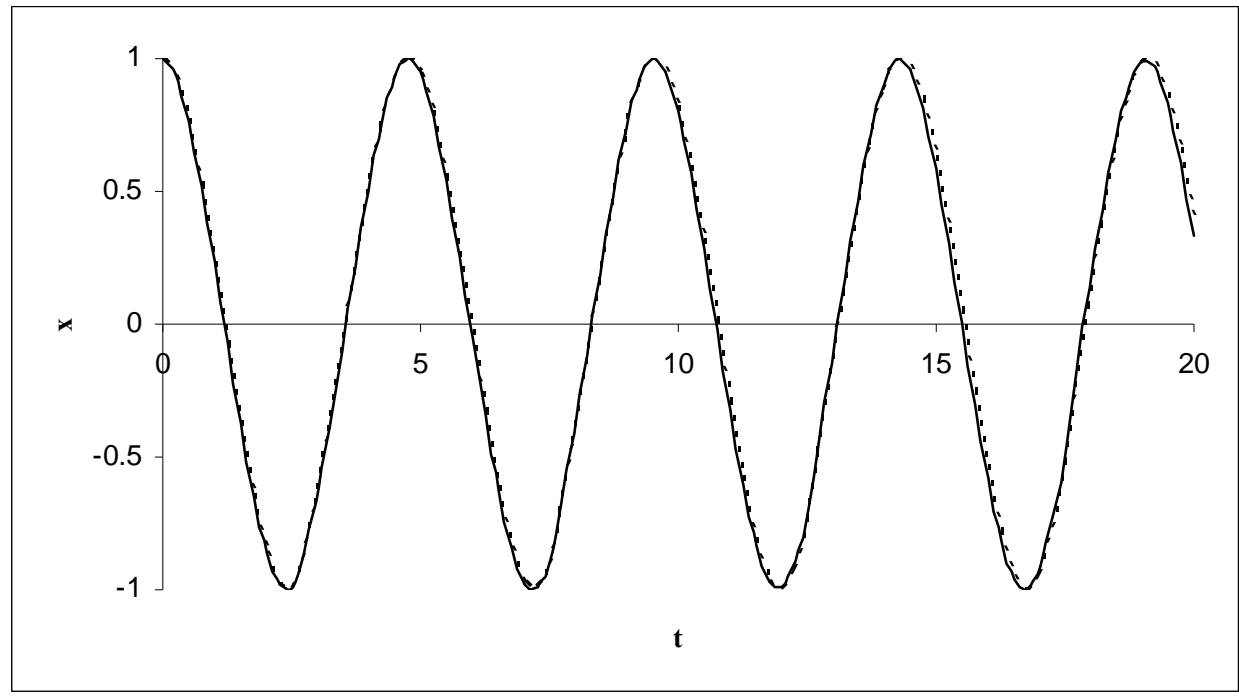

Fig.3. (a) First order approximate solution (dotted lines) of Eq. (10) is compared with the corresponding numerical solution (solid line) obtained by Runge-Kutta fourth-order formula for $a_{0}=0.98304$, $\varphi_{0}=0.0, k=0.0, \omega=1.0, \varepsilon_{1}=0.75$ and $\varepsilon=1.0$.

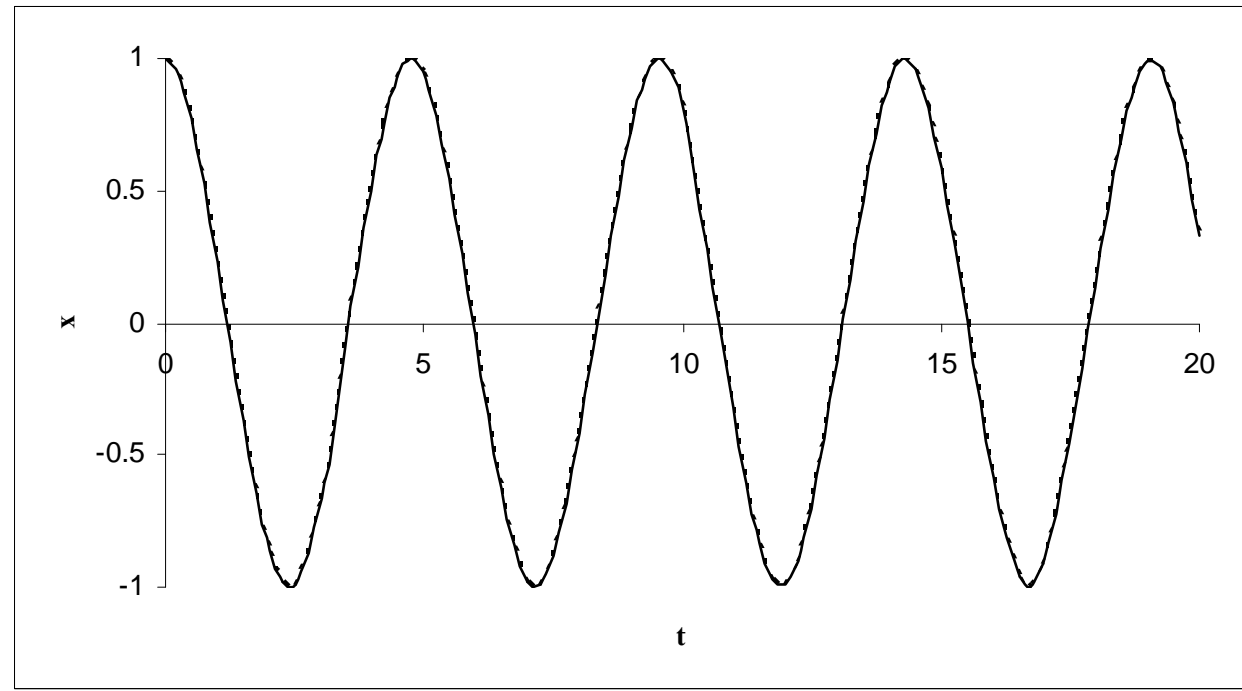

Fig.3. (b) Second order approximate solution (dotted lines) of Eq. (10) is compared with the corresponding numerical solution (solid line) obtained by Runge-Kutta fourth-order formula for $a_{0}=0.98164$, $\varphi_{0}=0.0, k=0.0, \omega=1.0, \varepsilon_{1}=0.75$ and $\varepsilon=1.0$. 


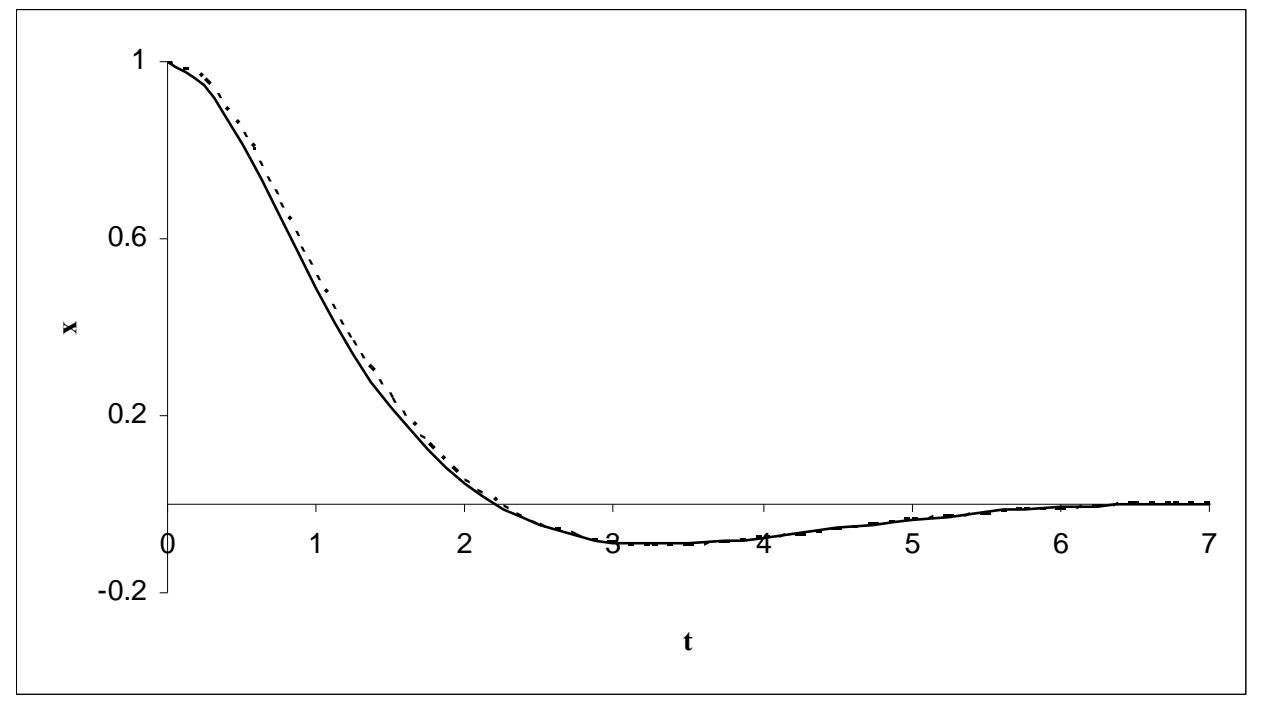

Fig.4. (a) First order approximate solution (dotted lines) of Eq. (10) is compared with the corresponding numerical solution (solid line) obtained by Runge-Kutta fourth-order formula for $a_{0}=1.06008$, $\varphi_{0}=-0.26899, k=\operatorname{sqrt}(.5), \omega=1.0, \varepsilon_{1}=0.0$ and $\varepsilon=1.0$.

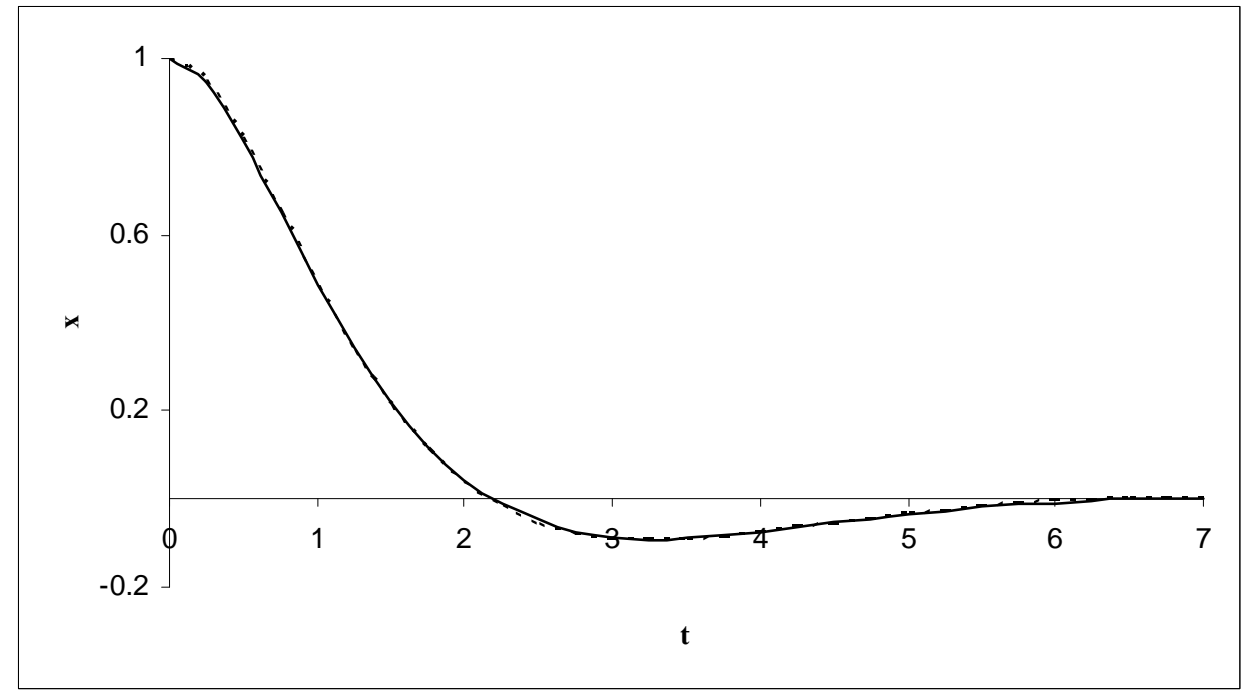

Fig.4. (b) Second order approximate solution (dotted lines) of Eq. (10) is compared with the corresponding numerical solution (solid line) obtained by Runge-Kutta fourth-order formula for $a_{0}=1.04049$, $\varphi_{0}=-0.25712, k=\operatorname{sqrt}(.5), \omega=1.0, \varepsilon_{1}=0.0$ and $\varepsilon=1.0$. 


\section{Conclusion}

In this paper, a new kind of analytical technique for a general second order nonlinear differential system with constant coefficients is presented. From the figures, it is clear to us that the first order approximate solutions continuously deviate from the numerical solutions with the increase of time $t$. Thus, we are forced to determine the second or higher order approximate solutions. The approximate solutions and the numerical solutions of Eq. (10) are obtained for the different damping effects and for several artificial constant with $\varepsilon=1.0$. Comparison is made between the solutions obtained by the homotopy perturbation method (dotted lines) and those obtained by the numerical procedure (solid line) in figures. This method shows effectively and accurately that large classes of second order approximate solutions converge rapidly to the numerical solutions in presence of significant damping effects with strong nonlinearity. Also this new homotopy perturbation technique is valid for strongly damped, weakly damped and undamped cases with strong nonlinearity. Moreover, it is also valid for weak nonlinearity of the systems. The variational equations are very important in a homotopy perturbation solution whatever the relations of them with $\varepsilon$. We conclude that, this new homotopy perturbation method is effective and accurate for nonlinear problems where the approximate solutions converge rapidly to the exact solutions. In a similar way, the method can be used to determine the higher order approximate solutions to the nonlinear systems.

\section{REFERENCES:}

1. Alam, M.S., Perturbation theory for nonlinear systems with large damping, Indian J. Pure appl. Math.32 (2001) 1453-1461.

2. Alam, M.S., A unified Krylov-Bogoliubov-Mitropolskii method for solving $n t h$ order nonlinear systems, J. Franklin Ins. 339 (2002) 239-248.

3. Alam, M.S., A unified Krylov-Bogoliubov-Mitropolskii method for solving $n t h$ order nonlinear systems with varying coefficients, J. Sound and Vibration 265 (2003) 987-1002.

4. Alam, M.S., A modified and compact form of Krylov-Bogoliubov-Mitropolskii unified KBM method for solving an $n t h$ order nonlinear differential equation, Int. J. Nonlinear Mech. 39 (2004)1343-1357.

5. Alam, M.S., Azad, M. A. K. and Hoque, M.A., A general Struble's technique for solving an $n t h$ order weakly non-linear differential system with damping, Int. J. of Non-linear Mechanics 41(2006) 905-918.

6. Bogoliubov, N.N. and Mitropolskii, Yu. A., Asymptotic Methods in the Theory of Nonlinear Oscillations, Gordan and Breach, New York, 1961.

7. Bojadziev, G. N., Damped nonlinear oscillations modeled by a 3-dimensional differential system, Acta Mechanica, 48 (1983) 193-201.

8. He, Ji-Huan, Modified Lindstedt-Poincare methods for some strongly non-linear oscillations, Part I: expansion of a constant, Int. J. of Nonlinear Mech. 37 (2002) 309-314.

9. He, Ji-Huan, The homotopy perturbation method for nonlinear oscillators with discontinuities, J. of Applied Mathematics and Computation 151 (2004) 287-292. 
10. Krylov, N.N. and Bogoliubov, N.N., Introduction to Nonlinear Mechanics, Princeton University Press, New Jersey, 1947.

11. Mendelson, K. S., Perturbation theory for damped nonlinear oscillations, J. Math. Physics.2 (1970) 3413-3415.

12. Murty, I.S.N., Deekshatulu,B. L. and Kirsna, G., On asymptotic method of Krylov-Bogoliubov for over damped nonlinear systems, J. Franklin Inst. 288 (1969) 49-64.

13. Murty, I. S. N., A unified Krylov-Bogoliubov method for solving second order nonlinear systems, Int. J. Nonlinear Mech. 6 (1971) 45-53.

14. Nayfeh, A. H., Perturbation methods, Wiley, New York, 1973.

15. Popov, I. P., A generalization of the Bogoliubov asymptotic methods in the theory of nonlinear oscillation (in Russian), Dokl. Akad. Nauk. SSSR 3(1956) 308-310

16. Struble, R. A., The geometry of the orbits of artificial satellites, Arch. Rational Mech. Anal. 7 (1961) $87-$ 104. 